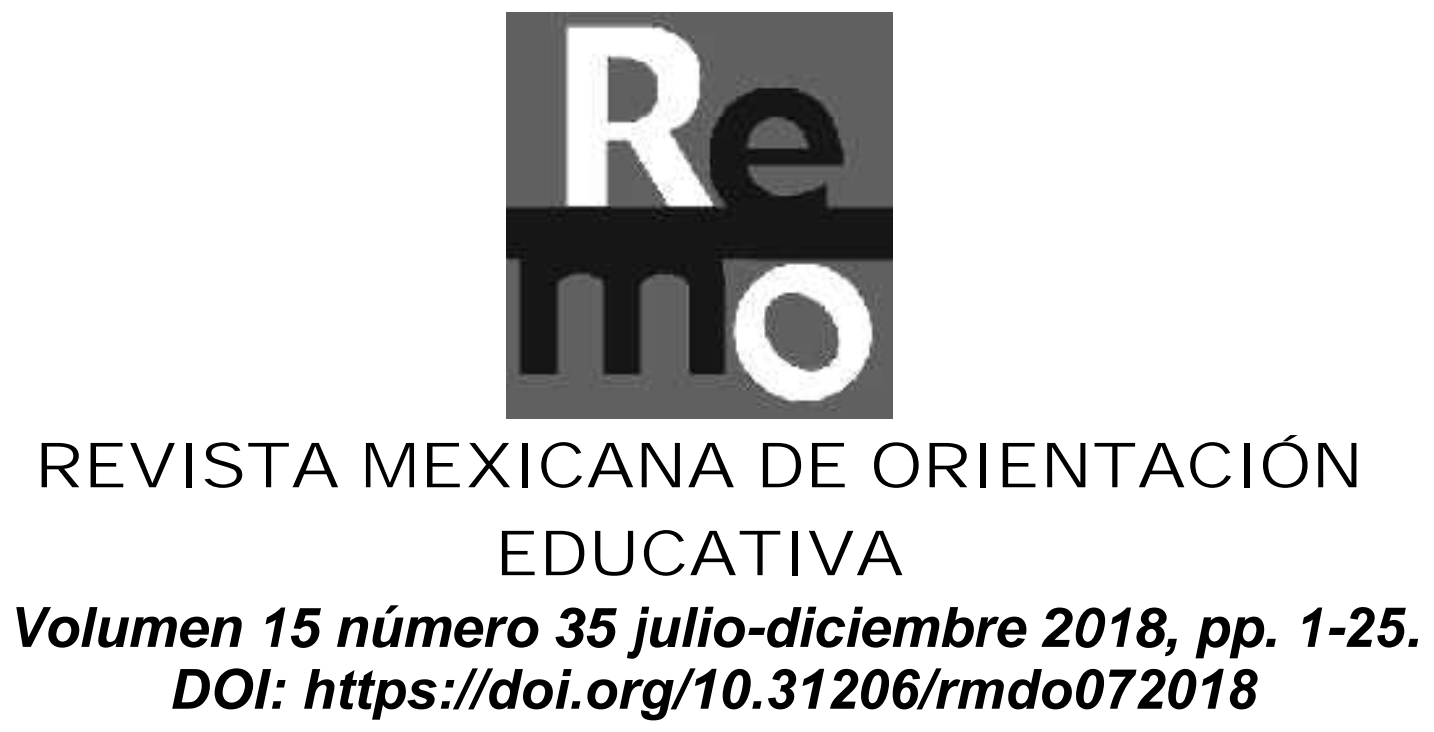

\title{
Factores asociados al rendimiento académico en estudiantes universitarios.
}

María Alicia Cano Celestino ${ }^{1}$

Rosalinda Robles Rivera ${ }^{2}$

Fecha de recepción 30 de mayo 2018

Fecha de aprobación 1 de octubre 2018

\footnotetext{
1 María Alicia Cano Celestino. Maestría en Psicología por la Facultad de Psicología de la Universidad Autónoma de San Luis Potosí (UASLP), Tesis doctoral por concluir. Labora en el Departamento de Orientación Educativa de la UASLP, y docente en la Facultad de Ciencias Químicas de la UASLP, Publicaciones en congresos, revistas de carácter nacional, e internacional. Correo electrónico psicologiaintegral_3@hotmail.com.

2 Rosalinda Robles Rivera. Maestría en Psicología por la Facultad de Psicología de la Universidad Autónoma de San Luis Potosí (UASLP), Aspirante al grado de Doctora en Educación. Especialidad en Orientación Educativa por la UASLP, Delegada de la Asociación Mexicana de Profesionales de la Orientación. Docente e Investigadora en las áreas de Psicología y Educación con énfasis en Orientación, Rendimiento Académico, Formación Docente, Motivación. Publicaciones en congresos, revistas de carácter nacional, e internacional, Correo electrónico rosyrobles07@hotmail.com.
} 
Resumen. El rendimiento académico ha sido abordado en múltiples investigaciones, a partir de ellas se ha identificado que los factores familiares, sociales, personales, escolares, de salud, laborales, entre otros, influyen de manera decisiva en el rendimiento académico de los alumnos universitarios. El presente estudio en proceso, tiene como el objetivo identificar factores asociados al rendimiento académico en estudiantes de la Facultad de Ciencias Químicas de la Universidad Autónoma de San Luis Potosí. Para tal efecto se emplean las pruebas; Terman-Merril, Valores interpersonales de Gordon y Adaptabilidad social de Moss.

Palabras clave: Rendimiento académico, alumnos universitarios, factores, variables endógenas y variables exógenas.

Abstrac. The academic performance has been approached in multiple investigations, from which it has been identified that the factors family, social, personal, school, Health, Labor, among others, influence in a decisive way in the academic performance of the University students. The present study in process, has as the objective to identify factors associated to the academic performance in students of the Faculty of Chemical Sciences of the Autonomous University of San Luis Potosí. For this purpose the tests are used; Terman-Merrill, Gordon's interpersonal values and social adaptability of Moss.

Key words: Academic performance, university students, factors, endogenous variables and exogenous variables.

Resumo. O desempenho acadêmico foi abordado em várias investigações, da qual foi identificado que os fatores familiares, sociais, pessoais, escolares, saúde, trabalho, entre outros, influenciam de forma decisiva no desempenho acadêmico da Estudantes universitários. O presente estudo em andamento tem como objetivo identificar fatores associados ao desempenho acadêmico em alunos da faculdade de ciências químicas da Universidade Autônoma de San Luis Potosí. Para o efeito, os ensaios são utilizados; Terman-Merrill, os valores interpessoais de Gordon e a adaptabilidade social do musgo. Palavras-chave: desempenho acadêmico, estudantes universitários, fatores, variáveis endógenas e variáveis exógenas. 


\section{Introducción}

El estudio de una carrera profesional exige a los alumnos diversas responsabilidades entre ellas; la inversión de largas jornadas de trabajo escolar dentro y fuera de la institución, el cumplimiento de diversas tareas y actividades para cada una de las materias, el desempeño de prácticas de laboratorio, profesionales y/o de servicio social, todo con una sola finalidad; la obtención de resultados que favorezcan el llevar a buen término los estudios profesionales.

Es decir, el rendimiento académico del alumno debe reflejarse como resultado de las acciones que demanda la institución educativa y el proceso de enseñanza-aprendizaje, que desde el enfoque pedagógico prevalece y se fundamenta con autores clásicos como Gimeno $(1976,16)$, quien después de una amplia investigación empírica, postula que: "las aptitudes, actitudes, aspiraciones, intereses personales... del hombre vienen condicionados por las influencias de su medio ambiente y que el entorno socio-cultural del alumno condiciona sus logros en la educación que imparten los establecimientos escolares y que se pueden evidenciar".

Lo anterior implica una palpable y transparente realidad, para entender el rendimiento académico como un producto condicionado por múltiples variables, factores y circunstancias a las que nos deberemos acercar, no solo en su proceso sino en la construcción de diseños y estrategias que enfocadas a un mejor resultado, cómo señalan Díaz Barriga y Hernández (2005) son los que permitirán controlar o seleccionar la información que le dispone aprender contenidos en contextos diversos y experienciales (Díaz-Barriga y Hernández, 2005) y como tal, lo más relevante, aprender a aprenderlos. Esto significa para la Educación Superior, centrarse en el desarrollo de competencias esenciales que permitan a las personas valerse por sí mismas, para intervenir y adaptarse a la sociedad.

La formación universitaria por tanto, deberá proporcionar a los alumnos los conocimientos teóricos y prácticos que permitan construirse un cimiento de competencias que contribuyan a su inserción al mercado de trabajo y al desarrollo social. Desde esta perspectiva la Universidad Autónoma de San Luis Potosí (UASLP), conforme al su Plan de Desarrollo Educativo 2015-2018 habrá de fomentar la educación integral y para lograrlo se puede apoyar en la teoría del Desarrollo Humano que tiene como principal 
tarea potenciar la esfera biopsicosocial del yo. Conforme a González (2000) el yo biopsicosocial abarca tres dimensiones: físico, psíquico y social.

Conforme a Martínez Carpio (2013: 14) la competencia desde la perspectiva del desarrollo humano "es una construcción social, donde se evidencia que la persona ha aprendido de forma significativa. Esto se expresa en el desarrollo de nuevos conocimientos, habilidades, y actitudes; integradas en capacidades y valores, fácilmente transferibles a diferentes situaciones de la vida y al desempeño profesional. Dentro de este proceso son esenciales el desarrollo de capacidades metacognitivas y la perspectiva clara del proyecto de vida de la persona".

Estas expresiones dan a conocer la preocupación de las autoridades por incidir en una formación más integral para los alumnos. El aprendizaje significativo conforme a Ausubel (1990) "es un proceso en el que ideas expresadas simbólicamente son relacionadas de modo no arbitrario y sustancial, no al pie de la letra, con lo que el alumno ya sabe... (con una imagen, un símbolo, un concepto, o una proposición). Es decir, el aprendizaje se vuelve significativo cuando el estudiante relaciona el material nuevo con la experiencia previa, por lo que la estructura cognoscitiva es un factor preponderante ya que es la base sobre la cual se desarrollará el conocimiento ulterior.

Es así como el aprendizaje, se inserta en los planes y programas de las universidades del País, para fortalecer al interior de las aulas un mejor rendimiento académico que impacte en los procesos de certificación para la calidad educativa, sin embargo hay otros aspectos que también deben considerarse como; la reprobación, el rezago académico, el abandono escolar, como indicadores que en su momento den el resultado esperado para cumplir no solo con los planes y programas, sino también con las políticas y criterios internacionales que demanda la sociedad actual (OCDE, PISA, CENEVAL, entre otros).

\section{Desarrollo}

El problema científico que atiende esta investigación es la persistencia de bajo rendimiento académico en las cinco carreras de la Facultad de Ciencias Químicas de la Universidad Autónoma de San Luis Potosí y se manifiesta con mayor incidencia en el curso propedéutico, lo que contribuye a la reprobación, rezago y deserción escolar. Por 
lo tanto, el tema de investigación se centra en la identificación de los factores asociados al bajo rendimiento académico en estudiantes universitarios.

El campo de investigación se circunscribe a los alumnos de nuevo ingreso a la Facultad de Ciencias Químicas de la Universidad Autónoma de San Luis Potosí, mismos que se dividen en dos grupos: Curso Propedéutico y Grupo de Primer semestre.

- Primer semestre. Se conforma por alumnos que aprobaron los exámenes de admisión, estos son psicométrico que representa el 15\% de la calificación total, el de examen de conocimientos que equivale al $45 \%$ y por último el EXANI-II del CENEVAL que representa el $40 \%$.

El Psicométrico consta de un total de 158 preguntas, distribuidas de la siguiente manera: 50 de razonamiento verbal, 18 reactivos de comprensión lectora y 90 de razonamiento abstracto, (Universidad Autónoma de San Luis Potosí, 2016).

El examen de conocimientos es elaborado por la UASLP y se destacan los contenidos de Química, Física, Matemáticas, Biología y Lenguaje y Conocimiento. EI EXANI-II del CENEVAL. La presente prueba se integra por dos instrumentos: Admisión y Diagnóstico. El examen de admisión mide la potencialidad para lograr nuevos aprendizajes y su objetivo es evaluar habilidades y conocimientos que son referente del buen desempeño de los aspirantes en el siguiente nivel educativo.

El examen diagnóstico por su parte tiene como objetivo evaluar los conocimientos representativos y relevantes de áreas contenidas en programas de estudios de la educación media superior (Centro Nacional de Evaluación para la Educación Superior, 2018).

- Curso Propedéutico. Va dirigido a los alumnos que obtuvieron bajas calificaciones en los exámenes de admisión; de conocimientos de la UASLP y EXANI-II del CENEVAL. El Reglamento interno de la Facultad de Ciencias Químicas (2010), establece en el Artículo 139 que el semestre propedéutico tiene como objetivo mejorar el nivel académico de los aspirantes a la licenciatura y no tiene validez curricular.

Las materias que se cursan en este semestre son: matemáticas, física, química con cinco horas a la semana cada una, biología y taller de lectura y redacción 4 horas a la semana y taller de habilidades lógico-matemáticas con 1 hora a la semana. 
El objetivo de la investigación es identificar cuáles de los factores propuestos (endógenos y exógenos que inciden con mayor frecuencia en el rendimiento académico de los alumnos del curso propedéutico y generar una serie de propuestas que permitan atender el desarrollo de competencias requeridas para mejorar el rendimiento académico y una trayectoria escolar con mayor probabilidad de éxito, entendiendo por éxito la finalización de su carrera profesional es decir, la eficiencia terminal.

Las variables endógenas son aquellas que se encuentran en el propio sujeto, en sus cualidades, habilidades, intereses, potencialidades, autoestima, valores, capacidad de relación, limitaciones, defectos, etc., aquello que está y/o pertenece al propio sujeto.

En ésta Investigación las variables endógenas, son las habilidades cognitivas y las interpersonales:

Las habilidades cognitivas son las competencias mentales necesarias para resolver situaciones académicas (adquisición de conocimientos), afectivas (saber manejar la presión, la burla, etc.) y sociales (relacionarse efectivamente) presentes en la formación escolar. Dentro de este rubro a su vez se examinan diez áreas: información y conocimientos, comprensión, vocabulario, síntesis, aritmética, juicio práctico, analogías, ordenamiento de frases, clasificación, seriación, coeficiente intelectual y capacidad de aprendizaje.

Las habilidades interpersonales se traducen en la capacidad de interactuar positiva y constructivamente con los otros y con la finalidad de conseguir un objetivo educativo concreto. Es decir: Habilidad de supervisión, capacidad de decisión en las relaciones humanas, capcidad de evaluación de problemas interpersonales, habilidad para establecer relaciones interpersonales, sentido común y tacto en las relaciones interpersonales y capacidad de adaptación.

Las variables exógenas son aquellos estímulos que se encuentran fuera de la persona, están en el exterior, lo familiar, social, la pareja, los profesores, los amigos, la universidad y su espacio físico, la administración de la escuela, etc.

En este caso, se consideran las características psicosociales (factores internos que al conjugarse con lo externo pueden causan malestar físico, psíquico y social y pueden llegar a repercutir en el rendimiento académico), los factores institucionales y los pedagógicos. 
Se considera la relación con padres, la práctica de deportes, consumo de drogas ilegales, consumo de drogas legales entre ellas al alcohol, el tabaco, problemas de conducta de la alimentación, depresión y problemas con la pareja.

Los factores institucionales se relacionan con los aspectos que pueden ocasionar bajo desmpeño académico, para este estudio se tomaron opiniones de los alumnos en cuanto a: organización de los horarios dentro de la facultad, instalaciones apropiadas al tamaño de los grupos y requerimientos de la carrera, la normativa institucional y apoyos extraescolares como tutorías, regularización etc.

En los factores pedagógicos se observa la evaluación que los alumnos hacen de los profesores en cuanto a: tipo de evaluación, dominio de los contenidos de la materia, motivación hacia el estudiante, cumplimiento del programa, preparación de los temas, puntualidad y asistencia, enfoque a la profesión, comunicación con los alumnos y dinamismo en clase.

De acuerdo a Jiménez (2000:30) el rendimiento académico es "el nivel de conocimientos demostrado en un área o materia correspondiente con la norma de edad y nivel académico".

El autor se sugiere que el nivel de conocimientos debe demostrarse a través de una evaluación correspondiente a la edad, nivel y año académico. En ésta los alumnos deben evidenciar competencias que avalen el rendimiento académico correspondiente.

Otro concepto es el que se refiere al conjunto de medidas efectivas obtenidas por el individuo en determinadas actividades académicas, como respuesta a un proceso de instrucción o formación interpretable de acuerdo con objetivos o propósitos educativos antes fijados. En tal sentido, son indicadores de competencias aptitudinales, ligadas además a factores volitivos, afectivos y emotivos. Tales competencias reflejan el grado de logro de los objetivos establecidos en los programas oficiales de estudio (Norvaez, 1986).

Para Norvaez (1986) el rendimiento académico que puede tener un alumno es el resultado de objetivos y propósitos fijados con anterioridad y marcan el rumbo de las acciones que conducirán a su logro. Por lo que las actitudes y aptitudes cobran especial importancia pues al parecer son responsables del rendimiento académico efectivo. 
El rendimiento académico es el resultado de una serie de factores; la familia, el grupo de pares, posibilidades económicas, profesores, compañeros, motivación, interés o gusto por las actividades que desarrolla en las materias, el gusto por la carrera elegida, el desarrollo profesional, etc., convirtiéndose así en un fenómeno multicausal.

Aliaga y cols., (2001) estudiaron variables psicológicas relacionadas con el rendimiento académico en matemática y estadística en alumnos de primer y segundo año de la Facultad de Psicología en la Universidad Nacional Mayor de San Marcos, concluyendo que existe relación significativa con la motivación y las estrategias de aprendizaje.

Sánchez de Tagle-Herrera, Osronio-Castillo, Valadez-Nava, \& Heshiki-Nakandakari (2010: 25) trataron de identificar los rasgos de personalidad e inteligencia asociados al rendimiento académico en estudiantes de la carrera de Médico Cirujano para lo que se empleó el test 16 factores que plantea Cattell y para el cociente de inteligencia $(\mathrm{Cl})$ se utilizó el Test Factor "G" de Cattell.

Al Identificar el rendimiento académico, se utilizó el promedio de calificaciones al término del primer año escolar. Los resultados indican que el promedio de calificaciones fue mayor para los alumnos regulares en comparación con los no regulares. Al aplicar la prueba "T" se encontraron diferencias significativas en los rasgos de inteligencia, los alumnos regulares presentaron más alto puntaje. Otros factores en los que se encontraron diferencias fueron: actitud cognitiva, emotividad y conciencia. Entre las conclusiones se encontró que existen diferencias significativas en tres rasgos de personalidad entre los alumnos regulares e irregulares: actitud cognitiva, emotividad y conciencia.

Existe consenso en que la lista de las causas del fracaso o del éxito escolar es amplia, ya que va desde lo personal hasta lo sociocultural existiendo en la mayoría de las ocasiones una mezcla de factores personales y sociales (Osorio, Leobano, \& Navarro, 2007).

La influencia en el rendimiento académico es multifactorial, hay factores propios del estudiante (endógenos), y otros que pertenecen o se encuentran en el mundo circundante (exógenos). No actúan aisladamente, el rendimiento académico es el resultado de la acción recíproca de lo interno y lo externo (Porcel \& Dapozo, 2010). 
Algunos estudios similares, realizados en Colombia por Vélez Van, Roa (2005). Carrión (2002) en Cuba, Valle González, Núñez, Martínez Piñeror (1999) en la Universidad de la Coruña, en España y por Villalobos en la Universidad de Costa Rica, abordan el tema a partir de variables cognitivas emocionales y socioeconómicas, entre al igual que en esta investigación, se incluyen resultados del examen de ingreso a la Universidad, pruebas de aptitudes intelectuales, factores relacionados con el estado emocional hacia el estudio, calificaciones obtenidas, tipo de familia, interés vocacional, hábitos de estudio y nivel académico de los padres.

Concluyendo que los resultados se basan en aproximaciones metodológicas de tipo predictivo, que utilizan modelos de regresión múltiple, pocas veces complementados con modelos explicativos que dificultan su análisis integral y en los que prevalece un promedio de 45 al $55 \%$ de reprobación, por lo que se hace necesario describir a detalle sus características así como la revisión de los criterios de evaluación, sus estilos y ponderaciones (Castrejón Pérez, 1988).

En la figura 1 es posible visualizar resultados específicos de éste trabajo en los dos grupos de esta investigación, agrupando para cada caso (Endógenos y Exógenos) habilidades, valores y factores, con sus respectivas características. 


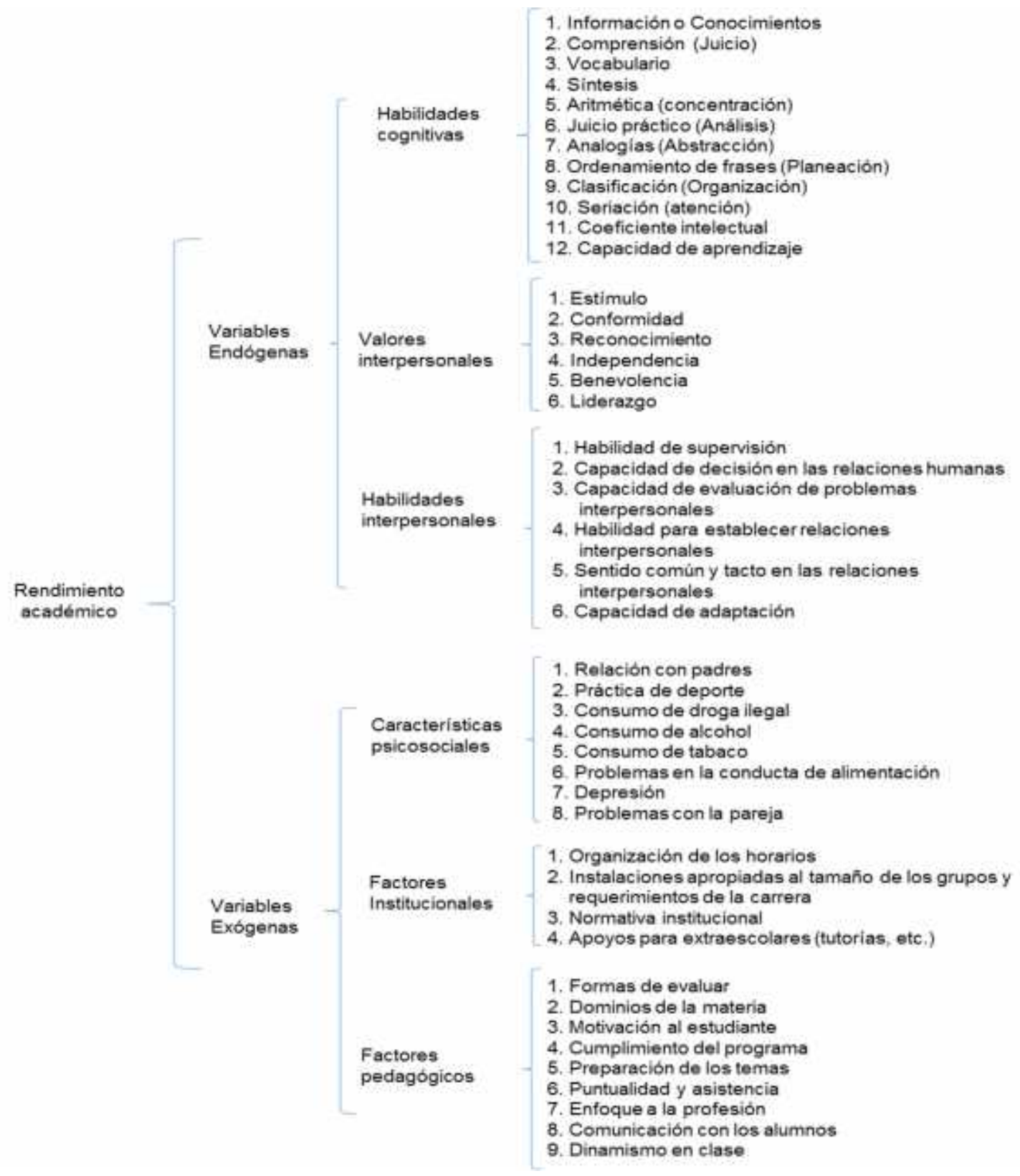

Figura 1. Factores que influyen en el rendimiento académico de los alumnos Universitarios. Diseño: María Alicia Cano Celestino.

\section{Metodología}

Es una investigación de enfoque mixto, descriptivo, de corte transversal y no experimental. Es enfoque mixto dado que se utilizaron métodos cuantitativos y cualitativos. De los métodos cuantitativos se emplea la recolección y análisis de datos con la aplicación de instrumentos psicométricos (pruebas y test). 
De las técnicas cualitativas se recurre a dos cuestionarios denominados factores psicosociales e institucionales, que identifican las opiniones sobre los factores que con mayor frecuencia inciden en el bajo rendimiento académico de los estudiantes.

Es descriptivo de corte transversal y no experimental. Para "Los estudios descriptivos buscan especificar las propiedades, las características y los perfiles de personas, grupos, comunidades, procesos, objetos o cualquier otro fenómeno que se someta a un análisis. Es decir, únicamente pretenden medir o recoger información de manera independiente o conjunta sobre los conceptos o las variables que se refieren, esto es, su objetivo no es indicar cómo se relacionan éstas" (Hernández Sampieri, Fernández Collado, \& Baptista Lucio, 2010, pág. 80).

Es transversal en tanto que se analiza a la población en un periodo corto de tiempo y no experimental en el sentido de que no se manipulan variables, por lo que se basa fundamentalmente en la observación de fenómenos tal y como se dan en su contexto natural para después analizarlos (Rosado 2003).

El test de Terman Merril, tiene como objetivo medir las principales habilidades que conforman la inteligencia (Carranza Heredia). El coeficiente mental del individuo (Cl), se obtiene de 10 sub- test que conforman la prueba a saber: Información o conocimientos, comprensión (Juicio), Vocabulario, Síntesis, Aritmética (Concentración), Juicio práctico (Análisis), Analogías (Abstracción), Ordenamiento de frases (Planeación), Clasificación (Organización) y Seriación (Atención). Este cuestionario de aptitud mental se empleó para evaluar la variable endógena denominada en este estudio habilidad cognitiva.

El test de adaptabilidad social MOSS, mide el grado en que una persona se comporta en situaciones que demandan supervisión y control de personal, además proporciona un índice general de la capacidad de adaptación que es capaz de mostrar en los diversos ambientes en los que se desenvuelve. Evalúa seis áreas: Habilidad de supervisión, Capacidad de decisión en las relaciones humanas, Capacidad de evaluación de problemas interpersonales, Habilidad para establecer relaciones interpersonales, Sentido común y tacto en las relaciones interpersonales y Capacidad de adaptación.

El test de valores interpersonales de Gordon evalúa valores para relacionarse con otras personas y adquieren relevancia en el ámbito personal, social, familiar y profesional 
del alumno y se conforma de seis valores: Estímulo, Conformidad, Reconocimiento, Independencia, Benevolencia y Liderazgo.

El cuestionario de factores institucionales está conformado por dieciséis preguntas, de las cuales siete se relacionan a la edad, género, semestre, carrera, fecha, ciclo escolar y el lugar que ocupa entre sus hermanos.

El resto de las preguntas se construyeron en escala tipo Likert nueve en total, y tienen el objetivo de indagar información relativa a los factores institucionales es decir, elementos presentes en la facultad y que influyen en el rendimiento académicos de los alumnos, entre ellos se encuentran; los horarios, capacitación del personal docente, aulas adecuadas, laboratorios de prácticas en condiciones para llevar a cabo las actividades demandadas, asesoría mediante tutorías, personal directivo con interés en las necesidades y desarrollo académico de los alumnos entre otras (ver anexo 4).

El cuestionario se aplicó a un total de 280 alumnos y sometió a la consistencia del Alpha de Cronbach que mide la fiabilidad de los instrumentos es decir, qué tan consistente es el resultado que proporciona. De esta forma el resultado para este cuestionario es un ALPHA 0.81, lo que indica que su consistencia es aceptable para la población en estudio, por lo que el resultado obtenido es confiable y válido.

El cuestionario de factores psicosociales consta de 29 preguntas. De ellas siete se refieren a datos como la edad, género, semestre, carrera, fecha, ciclo escolar y el lugar que ocupa entre sus hermanos.

Las 22 preguntas restantes en escala tipo Likert recogen la información con relación al padre, la madre, si existen problemas al interior de la familia, hace o no actividad física, el consumo de drogas legales e ilegales, explora si tiene o no pareja, y el tipo de relación que establece con ella en caso de tenerla, la conducta que tiene al interior del salón de clase, entre otras.

El cuestionario de factores psicosociales se aplicó a 280 alumnos y sometió a la prueba de consistencia en la que se obtuvo un Alpha de Cronbach de 0.88 , lo cual indica que es aceptable, por lo que el resultado es confiable y válido.

Los factores pedagógicos se encuentran constituidos por la opinión de los alumnos sobre el desempeño de los profesores en clase. Aquí se cuestiona respecto a: formas de evaluación, dominio de la materia, cumplimiento del programa, preparación de los 
temas, puntualidad y asistencia, enfoque a la profesión, comunicación con los alumnos y dinamismo en clase.

Esta evaluación se realiza en la facultad de Ciencias Químicas al término del semestre, como una condicionante para la inscripción al semestre siguiente. Los resultados de esta evaluación, no fueron proporcionados por la facultad, por lo que no se incluye información al respecto.

\section{Resultados}

El cien por ciento de la muestra está conformada por 280 alumnos, de ellos 112 es decir el 40 por ciento pertenecen al grupo del semestre Propedéutico, mientras que 168 alumnos o sea el 60 por ciento pertenecen al grupo de Primer semestre (ver figura 2).

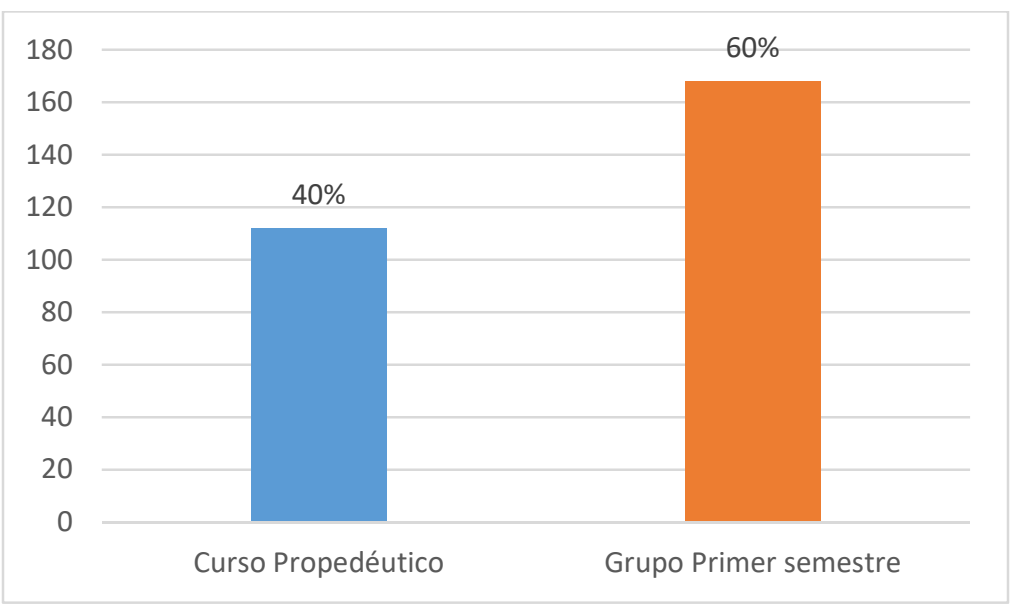

Figura 2. Número de participantes en el estudio de Factores que influyen en el rendimiento académico según el semestre de inscripción.

Del cien por ciento de la muestra el 63.3 por ciento son del género femenino, y el 37.6 por ciento son del género masculino.

Hacia el interior del grupo Propedéutico el 70 por ciento son de género femenino y el 30 por ciento corresponde al masculino. En el grupo de Primer semestre el 59 por ciento son de género femenino y el 41 por ciento corresponden al género masculino (ver figura 3). 


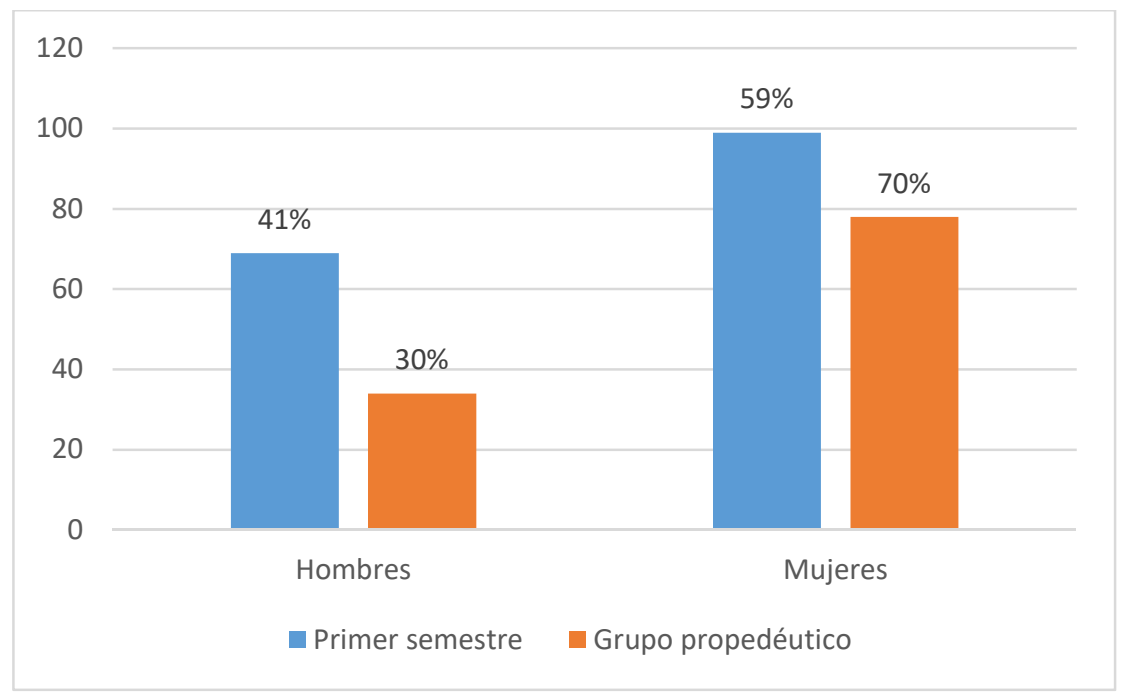

Figura 3. Género de los participantes en el estudio de Factores que influyen en el rendimiento académico

Como se observa a nivel general y por sub-grupos actualmente hay mayor participación de las mujeres en las aulas que en el pasado. Demostrando con esto el deseo de sobresalir y hacerlo en terrenos que antes eran exclusivos del hombre.

Las edad de los participantes oscila entre los 15 y 24 años, obteniéndose para ambos grupos la mayor frecuencia en los 18 años con el 53.5 por ciento para el grupo de primer semestre y el 31 por ciento para los alumnos del grupo propedéutico (ver figura 4).

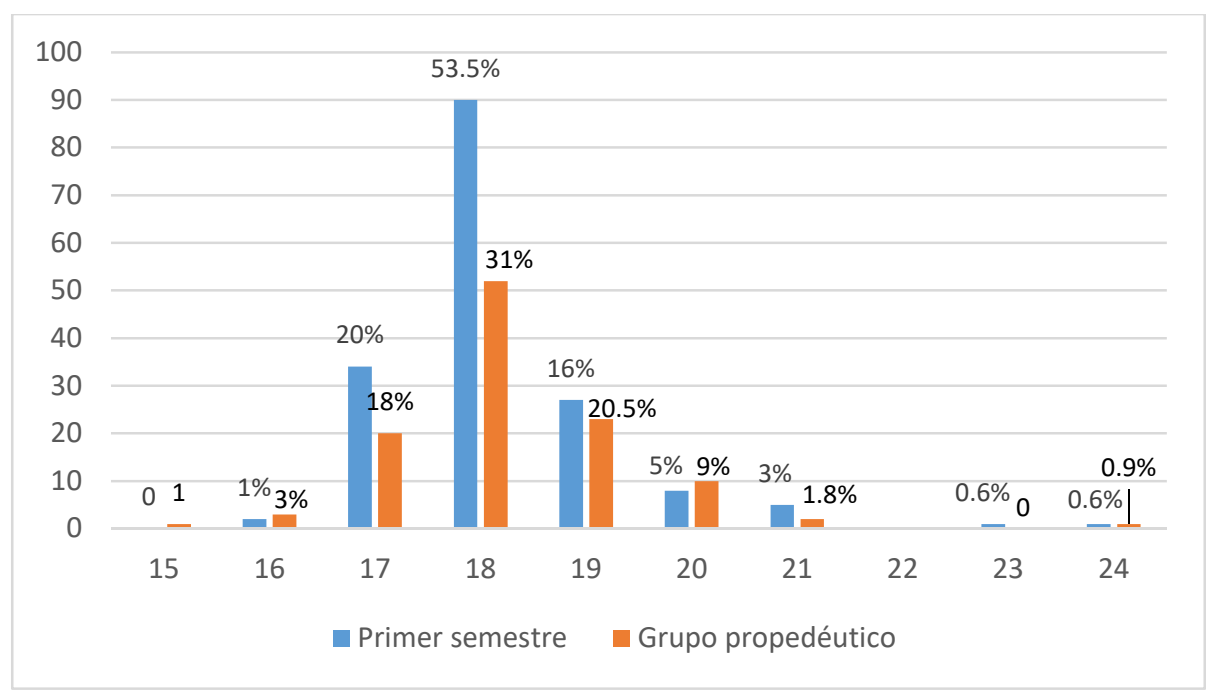

Figura 4. Edad de los alumnos participantes en el estudio Factores que inciden en el rendimiento académico. 
En cuanto a la carrera de elección la mayor frecuencia para el grupo de Primer semestre es Químico Farmacobiólogo con el 38 por ciento, mientras que para el grupo Propedéutico la Licenciatura en Ingeniería en Alimentos es la carrera con mayor inscripción con el 10 por ciento (ver figura 5).

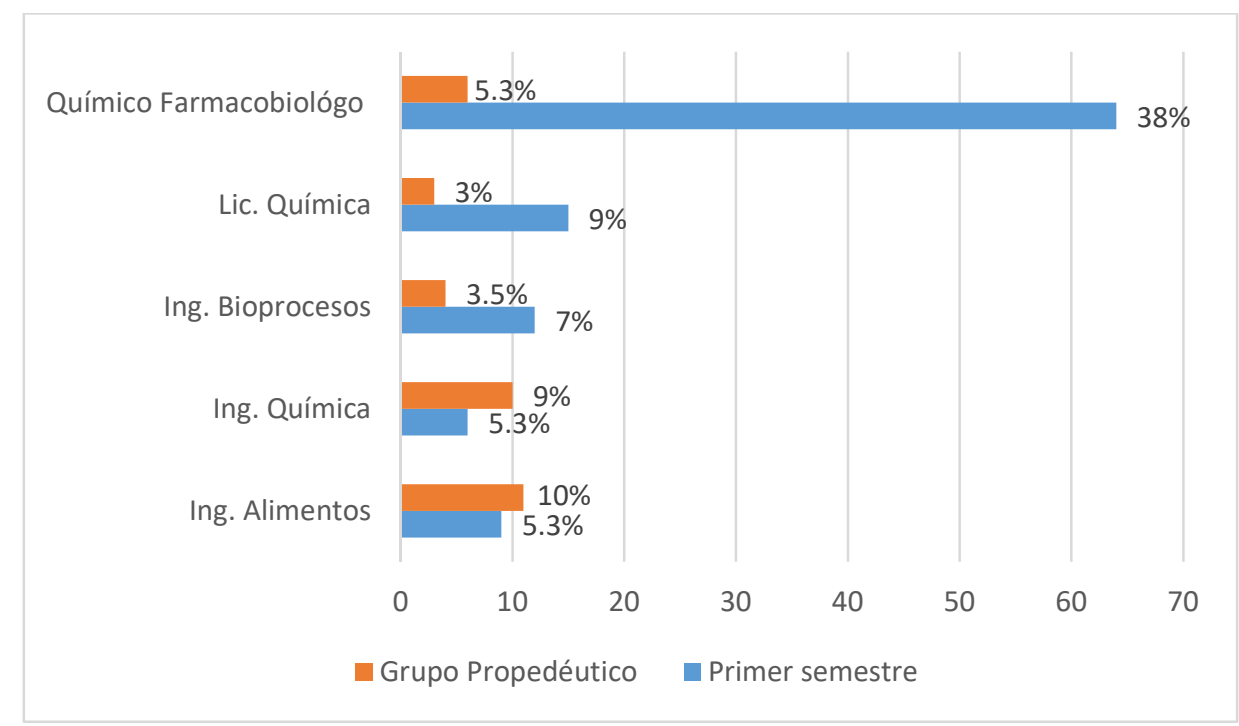

Figura 5. Inscripción a las carreras de la Facultad de Ciencias Químicas de la Universidad Autónoma de San Luis Potosí.

El Cuestionario de adaptabilidad social de MOSS, evalúa el grado de adaptación que una persona es capaz de establecer en diferentes situaciones y ámbitos de su vida y qué tan flexible puede ser para acoplarse a diferentes ambientes. De esta forma las áreas evaluadas por la prueba son: habilidad de supervisión, capacidad de decisión en las relaciones humanas, capacidad de evaluación de problemas interpersonales, habilidad para establecer relaciones interpersonales: capacidad para interactuar con personas, con una comunicación efectiva y una actitud positiva, poder entablar relaciones con las personas aunque sean desconocidas, sentido común y tacto en las relaciones interpersonales y capacidad de adaptación.

Los resultados en este test indican que el 61 por ciento de la población del grupo de Primer semestre y el 68 por ciento del grupo Propedéutico muestran bajos resultados en sentido común y tacto en las relaciones interpersonales (ver tabla 1 y 2 respectivamente). Además el grupo de Propedéutico tiene un índice general de adaptabilidad del 40 por 
ciento de la población en términos bajos. Esto refleja una diferencia significativa en el hecho de que los alumnos de propedéutico tienden a ser menos asertivos en sus relaciones, son ambiguos en la toma de decisiones y baja habilidad para escoger el momento, la situación y las palabras precisas para comunicar algo o realizar una acción. La adaptabilidad social es un indicativo importante para el desarrollo de los alumnos, puesto que durante su proceso de desarrollo académico se deben mover a diversos ambientes para la búsqueda de información, trabajar en equipos para el logro de ciertos objetivos, para el desarrollo de prácticas fuera de las aulas universitarias y el desarrollo de un servicio social, los alumnos que dificultad en esta área tenderán a ver limitado su aprendizaje y desarrollo.

Esta área hace referencia directamente a las variables endógenas, puesto que para poder adaptarse a los diversos ambientes se necesita de comprensión del ambiente en el que se desenvuelve, de un equilibrio emocional e intelectual que le permitan idear las estrategias necesarias para lograr esa adaptación.

\begin{tabular}{|c|c|c|c|c|c|c|c|}
\hline \multicolumn{8}{|c|}{ Se obtuvieron resultados de 168 alumnos } \\
\hline \multirow{2}{*}{ Rangos } & \multirow{2}{*}{ Bajo } & \multirow{2}{*}{$\begin{array}{l}\text { Inferior } \\
\text { al } \\
\text { promedio }\end{array}$} & \multicolumn{3}{|c|}{ Promedio } & \multirow{2}{*}{$\begin{array}{l}\text { Superior } \\
\text { al } \\
\text { promedio }\end{array}$} & \multirow{2}{*}{$\begin{array}{c}\text { Muy } \\
\text { superior }\end{array}$} \\
\hline & & & Bajo & Medio & Alto & & \\
\hline $\begin{array}{l}\text { Habilidad } \\
\text { de } \\
\text { supervisión }\end{array}$ & 14 & $\begin{array}{r}40 \% \\
54 \\
\end{array}$ & \multicolumn{3}{|c|}{94} & 6 & 0 \\
\hline $\begin{array}{l}\text { Capacidad de } \\
\text { decisiones en } \\
\text { relaciones } \\
\text { interpersonales }\end{array}$ & 1 & $\begin{array}{r}42 \% \\
\end{array}$ & \multicolumn{3}{|c|}{68} & 30 & 0 \\
\hline $\begin{array}{l}\text { Capacidad de } \\
\text { evaluación de } \\
\text { problemas } \\
\text { interpersonales }\end{array}$ & 5 & 34 & \multicolumn{3}{|c|}{122} & 7 & 0 \\
\hline $\begin{array}{l}\text { Habilidad para } \\
\text { establecer } \\
\text { relaciones } \\
\text { interpersonales }\end{array}$ & 0 & 44 & \multicolumn{3}{|c|}{55} & $\begin{array}{l}49 \\
41 \%\end{array}$ & 20 \\
\hline $\begin{array}{l}\text { Sentido común } \\
\text { y tacto en } \\
\text { relaciones } \\
\text { interpersonales }\end{array}$ & 30 & $61 \%_{72}$ & \multicolumn{3}{|c|}{65} & 1 & 0 \\
\hline $\begin{array}{l}\text { Capacidad } \\
\text { de } \\
\text { adaptación }\end{array}$ & 0 & 33 & 81 & 52 & 2 & 0 & 0 \\
\hline
\end{tabular}

Tabla 1. Resultados del Test de Adaptabilidad social (MOSS) grupo de Primer semestre. 


\begin{tabular}{|c|c|c|c|c|c|c|c|}
\hline \multicolumn{8}{|c|}{ Se obtuvieron resultados de 112 alumnos } \\
\hline \multirow{2}{*}{ Rangos } & \multirow{2}{*}{ Bajo } & \multirow{2}{*}{$\begin{array}{l}\text { Inferior } \\
\text { al } \\
\text { promedio }\end{array}$} & \multicolumn{3}{|c|}{ Promedio } & \multirow{2}{*}{$\begin{array}{l}\text { Superior } \\
\text { al } \\
\text { promedio }\end{array}$} & \multirow{2}{*}{$\begin{array}{c}\text { Muy } \\
\text { superior }\end{array}$} \\
\hline & & & Bajo & Medio & Alto & & \\
\hline $\begin{array}{l}\text { Habilidad } \\
\text { de } \\
\text { supervisión }\end{array}$ & 7 & 35 & & 68 & & 2 & 0 \\
\hline $\begin{array}{l}\text { Capacidad de } \\
\text { decisiones en } \\
\text { relaciones } \\
\text { interpersonales }\end{array}$ & 0 & $\begin{array}{r}47 \\
42 \%\end{array}$ & & 48 & & 17 & 0 \\
\hline $\begin{array}{l}\text { Capacidad de } \\
\text { evaluación de } \\
\text { problemas } \\
\text { interpersonales }\end{array}$ & 4 & $\begin{array}{r}46 \\
45 \%\end{array}$ & & 59 & & 3 & 0 \\
\hline $\begin{array}{l}\text { Habilidad para } \\
\text { establecer } \\
\text { relaciones } \\
\text { interpersonales }\end{array}$ & 1 & 39 & & 34 & & 30 & 8 \\
\hline $\begin{array}{l}\text { Sentido común } \\
\text { y tacto en } \\
\text { relaciones } \\
\text { interpersonales }\end{array}$ & 34 & $\begin{array}{r}42 \\
68 \%\end{array}$ & & 34 & & 2 & 0 \\
\hline $\begin{array}{c}\text { Capacidad } \\
\text { de } \\
\text { adaptación }\end{array}$ & 0 & $\begin{array}{r}45 \\
40 \%\end{array}$ & 45 & 22 & 0 & 0 & 0 \\
\hline
\end{tabular}

Tabla 2. Resultados del Test de Adaptabilidad social (MOSS) grupo Propedéutico.

El Cuestionario de valores interpersonales de Gordon, evalúa valores que son importantes para relacionarse con otras personas y adquieren relevancia en el ámbito personal, social, familiar y profesional del alumno. La prueba se conforma de seis valores: estímulo, conformidad, reconocimiento, independencia, benevolencia y liderazgo. Esta prueba indica que en ambos grupos el valor de benevolencia se encuentra con puntuaciones altas, para el curso de Primer semestre es del 58 por ciento, mientras que para el Propedéutico es el 64.5 por ciento (ver tabla 3 y 4 respectivamente). Las puntuaciones altas indican que los alumnos se muestran con una baja benevolencia lo que puede repercutir en su desarrollo no solo escolar si no también profesional. 


\begin{tabular}{|c|c|c|c|}
\hline \multicolumn{4}{|c|}{ Se obtuvieron resultados de 166 alumnos } \\
\hline Rangos & Bajo & Normal & Alto \\
\hline Est?mulo & $\begin{array}{c}79 \\
47 \%\end{array}$ & 66 & 21 \\
\hline Conformidad & 51 & 78 & 37 \\
\hline Reconocimiento & 44 & 59 & 63 \\
\hline Independencia & 16 & 82 & 68 \\
\hline Benevolencia & $58 \%$ & 66 & 3 \\
\hline Liderazgo & 17 & 78 & 71 \\
\hline
\end{tabular}

Tabla 3. Resultado de Valores interpersonales para el grupo de Primer semestre.

\begin{tabular}{|c|c|c|c|}
\hline \multicolumn{4}{|c|}{ Se obtuvieron resultados de 110 alumnos } \\
\hline Rangos & Bajo & Normal & Alto \\
\hline Est?mulo & 58 & 44 & 8 \\
\hline Conformidad & 37 & 51 & 22 \\
\hline Reconocimiento & 19 & 31 & 60 \\
\hline Independencia & 21 & 51 & 38 \\
\hline Benevolencia & $5 \% 11$ & 33 & 6 \\
\hline Liderazgo & 9 & 56 & 45 \\
\hline
\end{tabular}

Tabla 4. Resultado de Valores interpersonales para el grupo de curso Propedéutico.

El valor de benevolencia es mostrarse bueno y bondadoso con los otros, considerar importante hacer cosas por los demás y compartirlas con ellos, ayudar a los poco afortunados, ser generoso. Tomando en consideración este valor y cruzando la información con el valor de estímulo que se refiere a la necesidad de ser tratado con comprensión, amabilidad y respeto, y recibir apoyo de los demás se infiere que estos alumnos perciben poco apoyo de su ambiente externo, ven un medio social hostil, y con poca motivación para el logro de objetivos a esto se agrega el liderazgo en ambos grupos alto, muy posiblemente se refleje en una dirección negativa de los grupos en los que le corresponda trabajar, ya sea por asignación o por elección. Al respecto se debe tener presente que los estudiantes universitarios se preparan para incorporarse al termino de sus estudios a un mercado de trabajo en el que deberá demostrar sus competencias, sus conocimientos, habilidades, aptitudes y valores. Además de hacerse cargo de una responsabilidad social, lo cual contrasta con el resultado aquí obtenido.

Por lo tanto, este resultado sugiere que los factores endógenos son de vital importancia para el desarrollo académico de los alumnos y en particular en el rendimiento académico. Como ya se mencionó, los factores endógenos se encuentran en el propio sujeto, en sus 
cualidades, habilidades, intereses, potencialidades, autoestima, valores, capacidad de relación, limitaciones, defectos, etcétera.

Ingresar a la universidad y estudiar una carrera implica hacerse cargo de una responsabilidad social que poco se encuentra entre los estudiantes tal y como se refleja en el porcentaje logrado en ambos grupos de estudio.

La responsabilidad social del Universitario, como lo describen De la Calle Maldonado y Giménez Armentia (2011) es el compromiso personal con los demás y con el entorno que asume cada universitario hoy, para el día de mañana ejercer su profesión como un servicio a la sociedad en la búsqueda del bien común.

Las ideas centrales de la responsabilidad social universitaria conforme a las autoras antes mencionadas se relacionan con; el compromiso hacia los demás y con el entorno, descubrimiento personal de los valores, mayor conocimiento de la necesidad del otro y planteamiento del ejercicio profesional desde la búsqueda del bien común.

Conforme a la puntuación obtenida se concluye que los alumnos del curso Propedéutico presentan baja benevolencia y se puede ver plasmada en la poca responsabilidad hacia su desempeño escolar, así como en la baja actitud para el trabajo en equipo, pues consideran que el trabajo en solitario es más enriquecedor, lo hacen en tiempos disponibles conforme a sus necesidades y criterios de calidad.

Ver por el propio desarrollo, poseer una conducta egocéntrica opaca la posibilidad de interesarse por el desarrollo social y de aportar en beneficio del mismo.

En el Cuestionario de capacidad mental de Terman, se obtuvo para ambos grupos; grupos Primer semestre (ver tabla 5) y curso Propedéutico (tabla 6) resultados bajos en aritmética con el 25 por ciento para el primer grupo y el 60 por ciento para el segundo respectivamente. 


\begin{tabular}{|c|c|c|c|c|c|c|c|}
\hline \multicolumn{8}{|c|}{ Se obtuvieron resultados de 168 alumnos } \\
\hline Rangos & Muy Bajo & $\begin{array}{c}\text { Bajo } \\
\text { al } \\
\text { promedio }\end{array}$ & Suficiente & Satisfactor & Sobresaliente & $\begin{array}{c}\text { Muy sobresaliente } \\
\text { al } \\
\text { promedio }\end{array}$ & Excepcional \\
\hline $\begin{array}{c}\text { Información } \\
y \\
\text { conocimientos }\end{array}$ & 5 & 10 & 21 & 96 & 28 & 0 & 8 \\
\hline Comprensión & 0 & 0 & 4 & 32 & 50 & 52 & 30 \\
\hline $\begin{array}{c}\text { Significado } \\
\text { de } \\
\text { palabras }\end{array}$ & 0 & 4 & 38 & 84 & 18 & 14 & 10 \\
\hline $\begin{array}{l}\text { Selección } \\
\text { lógica }\end{array}$ & 0 & 2 & 14 & 115 & 29 & 7 & 1 \\
\hline Aritmética & $725 \%$ & 35 & 54 & 45 & 25 & 1 & 1 \\
\hline $\begin{array}{c}\text { Juicio } \\
\text { práctico }\end{array}$ & 7 & 12 & 33 & 52 & 35 & 22 & 7 \\
\hline Analogias & 0 & 0 & 13 & 72 & 48 & 35 & 0 \\
\hline $\begin{array}{c}\text { Ordenamiento } \\
\text { de } \\
\text { frases }\end{array}$ & 12 & 12 & 32 & 61 & 31 & 15 & 5 \\
\hline Clasificación & 2 & 4 & 36 & 100 & 17 & 8 & 1 \\
\hline Seriación & 0 & 5 & 23 & 62 & 32 & 32 & 14 \\
\hline
\end{tabular}

Tabla 5. Resultado de Test de Terman para los alumnos de Primer semestre.

\begin{tabular}{|c|c|c|c|c|c|c|c|}
\hline \multicolumn{8}{|c|}{ Se obtuvieron resultados de 112 alumnos } \\
\hline Rangos & Muy Bajo & $\begin{array}{c}\text { Bajo } \\
\text { al } \\
\text { promedio }\end{array}$ & Suficiente & Satisfactor & Sobresaliente & $\begin{array}{c}\text { Muy sobresaliente } \\
\text { al } \\
\text { promedio }\end{array}$ & Excepcional \\
\hline $\begin{array}{c}\text { Información } \\
y \\
\text { conocimientos }\end{array}$ & 11 & 13 & 12 & 62 & 14 & 0 & 0 \\
\hline Comprensión & 2 & 1 & 5 & 37 & 32 & 32 & 3 \\
\hline $\begin{array}{c}\text { Significado } \\
\text { de } \\
\text { palabras }\end{array}$ & 2 & 14 & 49 & 40 & 3 & 3 & 1 \\
\hline $\begin{array}{l}\text { Selección } \\
\text { lógica }\end{array}$ & 1 & 3 & 25 & so & 2 & 1 & 0 \\
\hline Aritmética & ${ }^{19} 60 \%$ & 48 & 28 & 15 & 2 & 0 & 0 \\
\hline $\begin{array}{c}\text { Juicio } \\
\text { práctico }\end{array}$ & 10 & 13 & 43 & 28 & 14 & 4 & 0 \\
\hline Analogias & 0 & 2 & 27 & 51 & 27 & 5 & 0 \\
\hline $\begin{array}{c}\text { Ordenamiento } \\
\text { de } \\
\text { frases }\end{array}$ & 12 & 11 & 39 & 33 & 11 & 5 & 1 \\
\hline Clasificación & 1 & 9 & 45 & 55 & 2 & 0 & 0 \\
\hline Seriación & 1 & 15 & 39 & 40 & 13 & 3 & 1 \\
\hline
\end{tabular}

Tabla 6. Resultado de Test de Terman para los alumnos de curso Propedéutico. 
La aritmética muestra los conocimientos numéricos del sujeto y su capacidad para manejar problemas o situaciones que demanden operaciones aritméticas, manejo de cantidades y problemas basados en matemáticas. Su importancia radica en que es un indicador del desarrollo cognoscitivo del sujeto, porque para que estos problemas sean resueltos correctamente se requiere que emplee su atención y concentración para comprender el problema, visualizarlo mentalmente y proponer las operaciones necesarias. Esta área está directamente relacionada con la capacidad de concentración.

\section{Conclusiones}

A partir de los resultados se puede inferir que las variables que inciden en el rendimiento académico de los alumnos son endógenas, el sentido común y el tacto, la benevolencia y el estímulo y la habilidad en aritmética, se relacionan directamente a la baja capacidad para la toma de decisiones, procesos de socialización y de trabajo en equipo, dificultando así el desarrollo académico con un fuerte impacto en el rendimiento escolar.

Las variables endógenas se encuentran en el propio alumno, en sus cualidades, habilidades, intereses, potencialidades, autoestima, valores, capacidad de relación, limitaciones, defectos, entre otras.

La facultad de Ciencias Químicas de la Universidad Autónoma de San Luis Potosí, deberá contemplar entre sus actividades formativas la posibilidad de incluir más contenidos de corte humano, aspectos de autoestima en el profesionista, trabajo en equipo, sentido social de las carreras, capacitación en inteligencia profesional, todo aquello que contribuya a una sólida identidad profesional. No se debe olvidar que la identidad profesional se logra únicamente se si tiene un sólido desarrollo personal ya que esta es la base para el desarrollo como profesionista.

Se deberá buscar la forma de equilibrar los contenidos académicos con desarrollo personal si desea que el rendimiento académico impere sobre la reprobación y la deserción escolar. 


\section{Bibliografía}

Aliaga Tovar, J., Ponce Díaz, C., Gutiérrez Olaya, V., Reyes Tejada, J., \& Pinto López, A. (2001). Variables psicológicasrelacionadas con el renidmiento académico en matemática y estadística en alumnos del primer y segundo año de la Facultad e Psicología de la UNMSM. Revista de Investigación en Psicología, 4(1), 1-18. Obtenido de http://revistasinvestigacion.unmsm.edu.pe/index.php/psico/article/view/5005/4070

Ardilla, A., \& Ostrosky-Solís, F. (2008). Desarrollo Histórico de las. Revista Neuropsicología, Neuropsiquiatría y Neurociencias, 1-21.

Ausubel, D. (1990). Psicología educativa. Un punto de vista cognoscitivo. México: Trillas.

Barriga, F. D. (2011). Experiencias educativas con recursos digitales. México: UNAM.

Carranza Heredia, B. G. (s.f.). Laboratorio de Desarrollo Organizacional. Obtenido de http://instdiagpsic. wikispaces.com/file/view/Instrumentos+para+el+Dx+Psic.+en+Prod.+y +Consumo.pdf.

Carrión Pérez, E. (2002). Validación de características al ingreso como predictores del rendimiento académico en la carrera de medicina. Educación Médica Superior, 1(16), 518. Obtenido de http://scielo.sld.cu/scielo.php?

Centro Nacional de Evaluación para la Educación Superior, A. (. (6 de Diciembre de 2018). Guía EXANI II. Examen Nacional de Ingreso a la Educación Superior. México: CENEVAL. Obtenido de http://www.ceneval.edu.mx/exani-ii

Definición de ABC. (2007). Obtenido de:

http://www.definicionabc.com/general/intelectual.php

Díaz, M., Escudero, T., Rodríguez, S., \& Vidal, G. (2002). Evaluación del rendimiento académico en la enseñanza superior. Comparación de resultados entre alumnos procedentes de la LOGSE y del COU. Revista de Investigación Educativa, 2(20), 357383. Obtenido de:

http://www.revistas.ucr.ac.cr/index.php/educacion/article/viewFile/1252/1315

Facultad de Ciencias Químicas de la Universidad Autónoma de San Luis Potosí. (Febrero del 2010). Reglamento Interno de la Facultad de Ciencias Químicas. San Luis Potosí.

Facultad de Ciencias Químicas, U. (3 de Junio de 2016). Facultad de Ciencias Químicas. Obtenido de UASLP: http://cienciasquimicas.uas/p.mx/index.php/facultad/comunidadacademica

Garbanzo Vargas, G. M. (2007). Factores asociados al rendimiento académico en estudiantes universitarios, una refexión desde la calidad de la educación superior pública. Educación, 31 ((1)), 46-63. Obtenido de:

http://www.revistas.ucr.ac.cr/index.php/educacion/article/view/1252 
García Linares, M., Casanova Arias, P., \& Pelegrina López, S. (2002). Los estilos educativos de los padres y la competencia académica de los adolescentes. Journal for the Study of Education and Development, 25(2), 147-168.

García López, I. R., Cuevas Salazar, O., Vales García, J. J., \& Cruz Medina, I. R. (2012). Impacto del Programa de Tutoría en el desempeño académico de los alumnos del Instituto Tecnológico de Sonora. Revista Electrónica de Investigación Educativa, 14(1), 106-121. Obtenido de http://redie.uabc.mx/redie/article/view/299

García Nuñez Del Arco, C. (2005). Habilidades sociales, clima social, familiar y rendimiento académico. Liberabit, 63-74.

Gordon, L. (2010). Manual Test SIV. Universidad San Pedro: Círculo de Estudio de Psicología. Obtenido de https://es.slideshare.net/carlabastos/test-devaloresinterpersonalessiv

Grinnell, R. M. (1997). Social work research \&evaluation: Quantitative and qualitative approaches. Itaca: Peacock Publishers.

Hernández Sampieri, R., Fernández Collado, C., \& Baptista Lucio, P. (2010). Metodología de la investigación. Chile: McGraww-Hill.

Jiménez, M. (2000). Competencia social: intervención preventiva en la escuela. .Infancia y Sociedad, 21-48.

Latiesa, M. (1992). La deserción universitaria,desarrollo de la escolaridad en la enseñanza superior. Éxitos y fracasos. Madrid, España. Obtenido de Centro de investigaciones sociológicas.

López-Vargas, O., Hederich-Martínez, C., \& Camargo-Uribe, Á. (2011). Estilo cognitivo y logro académico. Scielo , 13-25.

Marín, M., \& Infante, E. (2000). El fracaso académico en la Universidad: aspectos motivacionales e intereses profesionales. Revista latinoamericana de psicología.

Martínez Carpio, H. E. (2013). El enfoque por competencias desde la perspectiva del desarrollo humano. Aspectos básicos y diseño curricular. unifé, 9-22.

Montero Rojas, E., Villalobos Palma, J., \& Valverde Bermúdez, A. (2007). Factores institucionales, pedagógicos, psicosociales y sociodemográficos asociados al rendimieno académico en la ciudad de Costa Rica: Un análisis multinivel. Revista ELectrónica de Investigación Evaluación Educativa, 215-234.

Moreira Mora, T. E. (2009). Factores endógenos y exógenos asociados al renidmiento académico en matemática: un análisis multinivel. Educación, 61-80.

Norvaez, M. (1986). Psicología de la actividad escolar. México: Iberoamericana.

Osorio, M., Leobano, H., \& Navarro, J. (2007). Factores psicosociales que influyen en el éxito o fracso del aprovechamiento escolar en la asignatura de física básica. Caso del 
plantel Ignacio Ramírez Calzada. Espacios Públicos, 12(26). Obtenido de http://www.redalyc.org/articulo.oa?id=67612145014

Pérez L, L. E., Niño Ochoa, D. L., \& Páez Almanza, L. C. (2010). Actitudes, aptitudes y rendimiento académico en matemáticas. Actitudes, aptitudes y rendimiento académico en matemáticas (págs. 649-656). Colombia: CENGAGE Learning.

Pérez Sánchez, A., \& Castejón Costa, J. (1998). Un modelo casual-explicativo sobre la influencia de las variables psicosociales en el rendimiento académico. Bordón. Revista de Pedagogía, 50(2), 171-185. Obtenido de:

https://dialnet.unirioja.es/servlet/articulo?codigo $=54593$

Porcel, E. A., \& Dapozo, G. N. (2010). Predicción del rendimiento académico de alumnos. Revista Electrónica de Investigación Educativa., 12(2), 1-21.

Psicología. (27 abril de Abril de 2009). Obtenido de:

http://nipidea.blogia.com/2009/042701-las-7-aptitudes-mentales-de-thurstone..php

Quesada Oviedo, L. A. (10 de Marzo de 2000). Obtenido de:

https://www.google.com.mx/webhp?sourceid=chrome-instant\&ion=1\&espv=2\&ie=UTF$8 \# q=a p t i t u d+$ mental\&start $=10$

Rodríguez , S., Fita, E., \& Torrado , M. (06 de Junio de 2004). El rendimiento académico en la transición secundaria-universidad. Educación(334), 391-414. Obtenido de http://www.ince.mec.es/revistaeducacion/re334/re334_22.pdf

Rosado, M. Á. (2003). Metodología de investigación y evaluación. México: Trillas.

Sánchez de Tagle-Herrera, R., Osornio-Castillo, L., Valadez-Nava, S., \& HeshikiNakandakari, L. (2010). Rasgos de personalidad, inteligencia y rendimiento académico en estudiantes de la carrera de Médico Cirujano. Archivos en Médicina Familiar, 12(1), 25-31. Obtenido de http://www.medigraphic.com/pdfs/medfam/amf-2010/amf101d.pdf

SEP. (Diciembre de 2015). Dirección General de Planeación y Estadística Educativa. Obtenido de:

http://planeacion.sep.gob.mx/assets/images/principales_cifras/2014_2015_PRINCIPAL ES_CIFRAS_BOLSILLO.pdf

SEP. (Diciembre de 2015). Secretaría de Educación Pública. Obtenido de Dirección General de Planeación y Estadística Educativa:

http://planeacion.sep.gob.mx/assets/images/principales_cifras/2014_2015_PRINCIPAL ES_CIFRAS_BOLSILLO.pdf

SEP. (Diciembre. de 2015). Sistema Educativo de los Estados Unidos Mexicanos. Principales Cifras 2014-2015. Primera Edición. Obtenido de http://planeacion.sep.gob.mx/assets/images/principales_cifras/2014_2015_PRINCIPAL ES_CIFRAS_BOLSILLO.pdf

Sociedad de Psicología Aplicada. (1960). Prueba de Terman-Merril. Obtenido de: http://www.psico-system.com/2013/02/test-de-terman-merril.html 
Subuyuj Contreras, W. K. (Noviembre de 2013). Adaptabilidad social de un grupo de auditores que laboran en distintas empresas de la ciudad de Guatemala. Asunción, Guatemala. Obtenido de http://biblio3.url.edu.gt/Tesario/2013/05/43/Subuyuj-Wendy.pdf

Test de Ggordon, Desarrollo Humano Bicentenario. (s.f.). Obtenido de: https://desarrollohumanobicentenario.wordpress.com/tag/test-gordon/

Test de Moss. (s.f.). Obtenido de https://www.psic-system.com/2013/02/test-demoss.html

Test de valores de Gordon Allport-Psicología y Mente. (s.f.). Obtenido de: https://psicologiaymente.com/personalidad/test-estudio-valores-allport

UASLP. (Junio de 3 de 2016). Facultad de Ciencias Químicas. Obtenido de UASLP: http://cienciasquimicas.uaslp.mx/index.php/facultad/mision-vision

UASLP. (2016). Facultad de Ciencias Químicas. Obtenido de Universidad Autónoma de San Luis Potosí: http://cienciasquimicas.uaslp.mx/index.php/facultad/acerca-de

UASLP. (3 de Junio de 2016). Universidad Autónoma de San Luis Potosí. Obtenido de UASLP: http://www.uas/p.mx/universidad/historia/a\%C3\%B1o-de-1923

UASLP. (3 de Junio de 2016). Universidad Autónoma de San Luis Potosí. Obtenido de UASLP: http://www.uas/p.mx/Documents/Universidad/Sitio\%20rector/Informes/20142015/2014-2015-06-Indicadores-institucionales.pdf

Universidad Autónoma de San Luis Potosí. (2016). informe de Rrectoría 2017-2018. San Luis Potosí.

Vélez van Meerbeke, A., \& Roa González, N. (2005). Factores asociados al rendimiento académico en estudiantes de médicina. Educación Media, 8(2), 24-32. Obtenido de http://scielo.isciii.es/pdf/edu/v8n2/original1.pdf 